\title{
LA LIBS
}

\section{DANS L'INDUSTRIE NUCLÉAIRE}

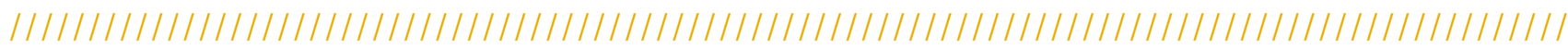

Jean-Baptiste SIRVEN ${ }^{1, *}$, Daniel L'HERMITE ${ }^{1}$, Jean-LuC LACOUR ${ }^{1}$, Céline QUERE ${ }^{1}$, Thomas VERCOUTER ${ }^{1}$

${ }^{1}$ Université Paris-Saclay, CEA, Service d'Etudes Analytiques et de Réactivité des Surfaces, 91191 Gif-sur-Yvette, France

* jean-baptiste.sirven@cea.fr

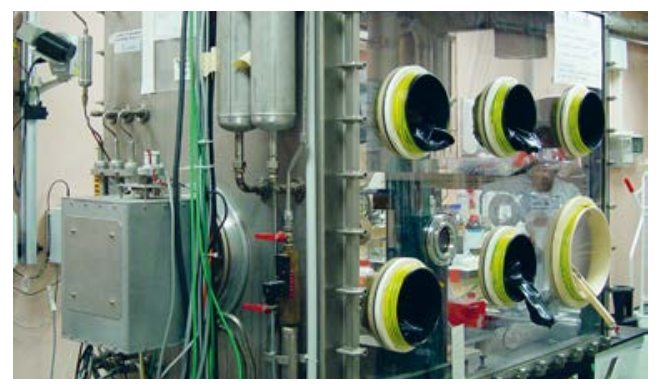

\begin{abstract}
La technique LIBS présente des avantages uniques pour réaliser des mesures élémentaires en environnement difficile. Elle est développée en France et dans d'autres pays pour répondre à de nombreux besoins de la filière électronucléaire. Elle nécessite pour cela une instrumentation variée capable de s'adapter aux diverses problématiques analytiques, configurations et contraintes de mise en œuvre propres au secteur nucléaire.
\end{abstract}

https://doi.org/10.1051/photon/202010330

Article publié en accès libre sous les conditions définies par la licence Creative Commons Attribution License CC-BY (https://creativecommons.org/licenses/by/4.0), qui autorise sans restrictions l'utilisation, la diffusion, et la reproduction sur quelque support que ce soit, sous réserve de citation correcte de la publication originale.

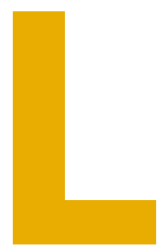

a France produit plus de la moitié de son énergie électrique par la filière nucléaire. Secteur hautement technologique, le nucléaire présente des enjeux majeurs au niveau opérationnel : garantir le fonctionnement sûr des centrales, sécuriser la production, recycler le combustible, gérer les déchets, et bientôt le démantèlement des installations. Au niveau de la recherche, l'industrie nucléaire a toujours été un moteur de l'innovation, soit pour répondre aux besoins opérationnels, soit pour mettre au point les technologies du futur.

En particulier, l'analyse physicochimique est essentielle à toutes les étapes, qu'il s'agisse de la fabrication des matériaux, du contrôle de procédé, de la caractérisation des déchets ou des composants en fin de vie. Orientée par les besoins des industriels, la R\&D doit répondre aux exigences de performance technico-économique, de prise de décision rapide et efficace, aux contraintes techniques et réglementaires en matière de sûreté / sécurité, ou encore de limitation de l'impact environnemental de l'activité. C'est pourquoi les techniques mises en œuvre doivent être instrumentalement robustes pour pouvoir être utilisées de façon intensive, éventuellement en ligne ou in situ, avec des systèmes compacts et nécessitant le moins de maintenance et la plus aisée possible. Et elles doivent, bien sûr, répondre au besoin analytique en termes de représentativité, de rapidité, de sensibilité, de justesse et de reproductibilité de la mesure, parmi d'autres critères.
Les techniques optiques basées sur l'interaction lumière-matière et la spectroscopie sont particulièrement bien adaptées pour cela. Parmi elles, la spectroscopie de plasma produit par ablation laser (laser-induced breakdown spectroscopy ou LIBS) présente l'avantage majeur de coupler en un même processus léchantillonnage du matériau et son excitation. C'est une technique privilégiée pour l'analyse élémentaire en conditions industrielles, en ligne, de terrain ou en environnement difficile. Elle est développée principalement pour l'analyse des solides, et dans une moindre mesure des liquides et des aérosols. Elle a pris son essor en France dans les années 1980 grâce aux travaux de l'équipe de $P$. Mauchien au CEA, et il est intéressant de noter que les premières études sur 
le sujet étaient liées à l'énergie nucléaire. Elle s'est ensuite largement développée au plan national dans différents domaines, à l'image de son évolution rapide à l'échelle mondiale à partir des années 2000.

Cet article présente un état de l'art de l'instrumentation et des applications de la LIBS dans l'industrie nucléaire, avec un focus particulier sur la contribution française dans ce domaine. On précise que l'on se limite ici aux travaux liés à la production délectricité et que les autres aspects du nucléaire ne seront pas abordés (dissuasion, non-prolifération, criminalistique, NRBC, fusion).

\section{UNE INSTRUMENTATION SPÉCIFIQUE}

En raison de son caractère tout optique, la technique est utilisable à distance, ce qui permet d'envisager des mesures directes d'échantillons radioactifs en enceinte confinée (boîte à gants voire chaîne blindée). On peut ainsi conserver l'instrumentation en dehors de la zone radioactive, et focaliser le faisceau laser à travers un hublot avec une distance de l'ordre du mètre, ou davantage si nécessaire. Ceci améliore considérablement les conditions d'utilisation et de maintenance du système en environnement nucléaire. De plus, les mesures LIBS sont réalisables sans préparation, ce qui autorise une analyse directe sans manipulation de l'échantillon et limite l'exposition des opérateurs à la radioactivité et la production de déchets supplémentaires. La LIBS se positionne ainsi en complément de techniques d'analyse élémentaire plus conventionnelles telles que l'ICP-OES ou l'ICP-MS par exemple. Ces dernières sont en général analytiquement plus performantes mais elles nécessitent un prélèvement et une mise en solution préalable des échantillons solides.

D'autres configurations instrumentales plus intrusives sont pertinentes pour des mesures en milieu radioactif. En particulier, les systèmes LIBS compacts, susceptibles d'être télémanipulés ou embarqués sur un robot d'inspection, présentent l'avantage de pouvoir venir au contact de surfaces inaccessibles en vision directe par un système à distance. Les systèmes portables de type « pistolet », largement développés au plan commercial depuis 2013, présentent toutefois quelques inconvénients potentiels : la résistance aux radiations, et les performances du système compact de détection (spectromètre et détecteur), dont la résolution spectrale et temporelle est généralement limitée. Une alternative consiste à déporter l'analyse en injectant l'impulsion laser dans une fibre optique. La collecte de l'émission du plasma étant elle aussi récupérée par fibre optique, on peut concevoir une sonde très compacte avec un rayon d'action de plusieurs mètres voire plusieurs dizaines de mètres. Cette option permet de conserver toute liberté sur le choix du spectromètre et du détecteur. En contrepartie, l'énergie

\section{SPIE.PHOTONEX}

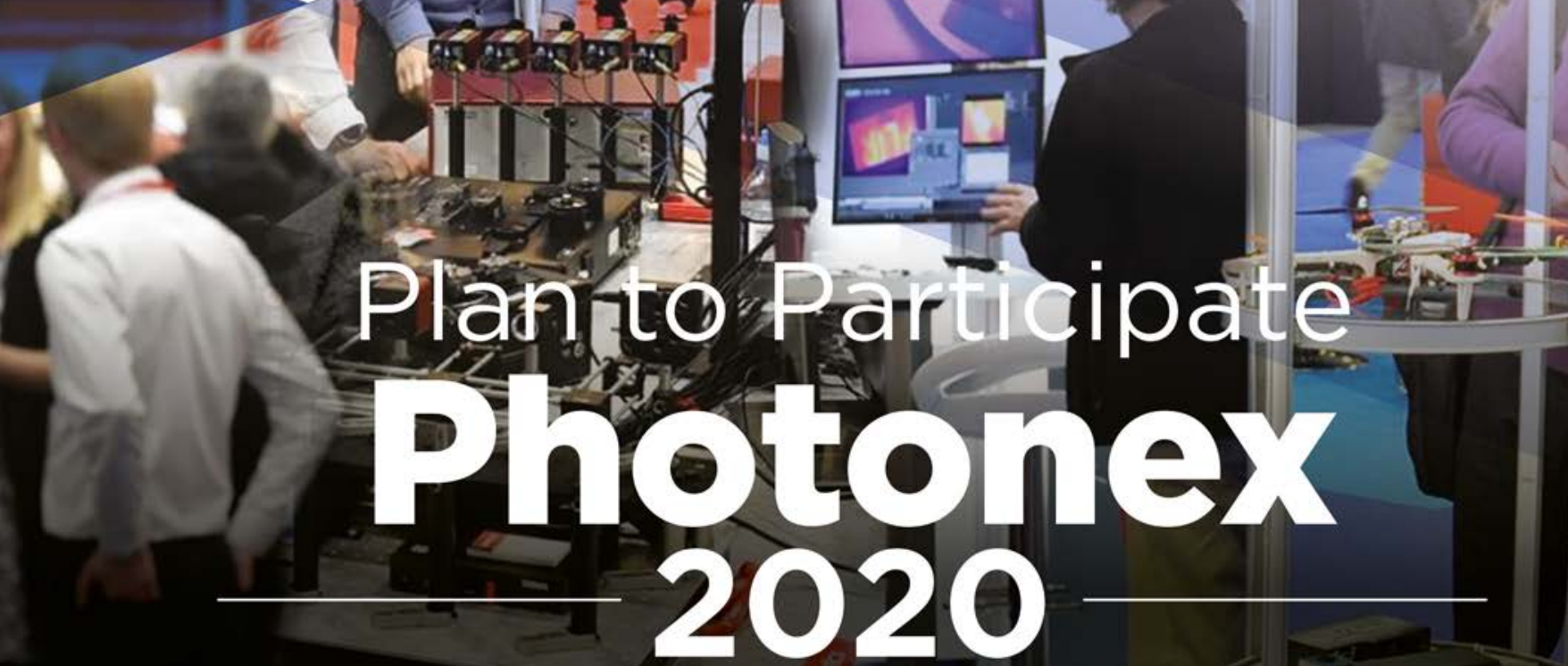

An online forum featuring photonics, imaging, lasers, and optical technologies-from pure research to development of user solutions. 


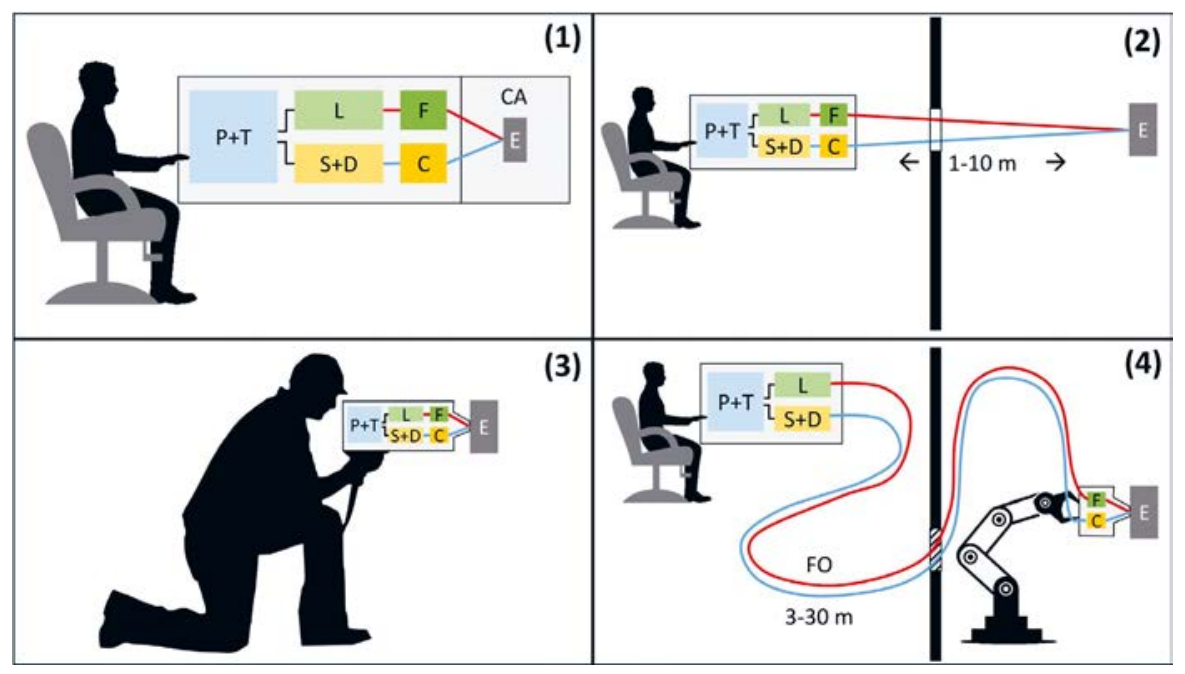

de l'impulsion laser transmise par la fibre est limitée, et la divergence du faisceau en sortie est importante. Il est donc difficile d'atteindre un éclairement élevé sur l'échantillon, et la tolérance sur la position de la surface par rapport au plan focal est faible.

Les potentialités de la LIBS en contexte nucléaire ont donc été identifiées très tôt, et elles sont multiples. Cette versatilité limite cependant la standardisation de l'instrumentation et des méthodes analytiques. Ainsi, les systèmes et les protocoles de mesure doivent être adaptés en fonction des contraintes de mise en œuvre et des objectifs analytiques. En particulier, la robustesse et la nucléarisation des instruments sont un enjeu majeur. Un autre point dur particulier au domaine nucléaire est l'analyse quantitative de matériaux pour lesquels il n'existe pas ou peu d'étalons ou d'échantillons de référence.

\section{ÉTAT DE L'ART EN FRANCE}

Les premiers travaux sur la LIBS en France réalisés par le CEA dans la seconde moitié de la décennie 1980 ont porté sur la vitrification des déchets. Une coulée de verre non radioactif produite par une installation pilote a été analysée à quelques mètres de distance en utilisant un laser $\mathrm{XeCl}$ et un spectromètre de haute résolution. Les limites de détection des éléments mesurés étaient dans la gamme ppm. Malgré les limites de la technologie
Figure 1. Quatre configurations de systèmes LIBS : (1) instrumentation de laboratoire ou de micro-analyse, (2) analyse à distance, (3) système portable, (4) système fibré. L : laser. $S$ : spectromètre. $D$ : détecteur. $F$ : optique de focalisation. $C$ : optique de collecte. $\mathrm{E}$ : échantillon. $\mathrm{P}$ : pilotage. $\mathrm{T}$ : traitement. CA: chambre d'analyse. FO : fibres optiques.

de l'époque, notamment en matière de détecteur, les résultats analytiques obtenus étaient déjà de bon niveau. La LIBS est aujourd'hui utilisée comme technique standard de soutien analytique aux développements de procédés de vitrification.

Les travaux se sont ensuite orientés vers la cartographie élémentaire des solides par LIBS avec une résolution latérale à l'échelle micrométrique. Dans les années 1990, une résolution de $3 \mu \mathrm{m}$ a ainsi été démontrée sur des cartographies de 500 à $600 \mu \mathrm{m}$ de côté d'échantillons de combustible nucléaire simulé $\mathrm{UO}_{2} / \mathrm{CeO}_{2}$. Cette technique a ensuite été développée vers d'autres thématiques en lien avec les matériaux du nucléaire, notamment pour le stockage des déchets, et pour les alliages de zirconium des gaines de combustibles. Dans ce dernier cas, la cartographie quantitative de l'oxygène et de l'hydrogène a été réalisée avec une résolution latérale inégalée de $1 \mu \mathrm{m}$, et une limite de détection de 60 ppm pour l'hydrogène.
L'analyse quantitative par LIBS de matériaux nucléaires a également fait l'objet de recherches au CEA. On peut citer la mesure d'impuretés dans l'uranium et le plutonium, puis à partir des années 2000, les travaux sur les réacteurs de quatrième génération. La caractérisation des sels fondus en boîte à gants a été étudiée, ou encore la détection de particules dans les réacteurs à caloporteur hélium, ainsi que le suivi de la pureté chimique du caloporteur dans les réacteurs à sodium liquide, avec des limites de détection de quelques ppm pour des impuretés métalliques. Dans ce même contexte de surveillance des réacteurs, la détection d'aérosols de sodium issus d'une éventuelle fuite de canalisation a également fait l'objet de développements expérimentaux.

Enfin, mentionnons le secteur du démantèlement des installations, un domaine dans lequel les avantages de la LIBS en matière d'analyse rapide et de terrain peuvent être décisifs. Il s'agit ici principalement d'identification de matériaux, notamment pour en dresser l'inventaire avant les opérations de déconstruction, ou pour orienter le déchet vers la filière appropriée. D’autres études ont été réalisées pour caractériser certains composants peu accessibles et/ou situés dans des zones fortement irradiantes.

\section{À L'INTERNATIONAL}

À l'international, et pour les raisons que nous avons évoquées précédemment, la LIBS a également été développée dans le domaine électronucléaire. C'est aux États-Unis que les travaux sont les plus nombreux, avec des contributions importantes sur la caractérisation de combustibles, notamment irradiés et contenant des produits de fission. De façon plus marginale, on peut également signaler des études liées au retraitement du combustible, à la fragilisation des structures nucléaires, ou encore plus récemment aux réacteurs à sels fondus. De son côté, le Japon maintient 
une activité importante dans le domaine nucléaire, avec des études sur des systèmes fibrés pour le démantèlement du réacteur de Fukushima, et sur les alliages de zirconium des gaines de combustible. En Inde, le BARC mène des recherches depuis une dizaine d'années sur les combustibles au thorium et sur la vitrification du combustible usé. Au Royaume-Uni, plusieurs développements instrumentaux ont été réalisés dès les années 1990 pour effectuer des mesures in situ en réacteur au moyen de systèmes fibrés. Des études plus récentes portent sur le démantèlement des réacteurs au graphite, et sur le diagnostic de contamination de l'acier. Enfin, en Chine on relève depuis peu quelques travaux sur l'analyse des aciers de structure avec des systèmes fibrés.

Notons pour terminer une application de la LIBS particulièrement d'intérêt dans le domaine nucléaire : l'analyse isotopique. Celle-ci est possible si les raies des différents isotopes sont suffisamment séparées par rapport à la largeur des raies émises par le plasma. Depuis une dizaine d'années, on assiste à un regain d'intérêt pour cette thématique à travers le développement d'une technique dérivée de la LIBS baptisée LAMIS (laser ablation molecular isotopic spectrometry). Cette approche, basée sur l'émission moléculaire plutôt qu'atomique, permet de réaliser l'analyse isotopique principalement des éléments légers $(H, B, C, O \ldots)$.

\section{CONCLUSION}

La LIBS a été développée en France historiquement pour répondre à certains besoins de l'industrie électronucléaire. Par les contraintes qu'il impose et la diversité des problématiques analytiques que l'on y rencontre, ce domaine est un terrain privilégié pour la technique. Elle possède en effet plusieurs avantages indéniables par rapport aux techniques conventionnelles, notamment la possibilité d'analyse à distance, rapide et directe, la possibilité de réaliser des cartographies élémentaires de solides à l'échelle micrométrique, et de nombreuses configurations expérimentales susceptibles de s'adapter à des cas de figure très variés. En contrepartie, il est difficile d'atteindre un certain niveau de standardisation aussi bien de l'instrumentation que des méthodes analytiques, et cette difficulté est à la source de nombreux développements scientifiques et techniques. Dans cet article, nous avons évoqué de nombreux développements et applications, principalement $d u$ CEA, couvrant tous les aspects du nucléaire civil, de la fabrication du combustible à son traitement/recyclage. Et l'on peut penser que dans le futur, les thématiques du démantèlement des installations, de la surveillance des sites de stockage de déchets, ou encore des nouveaux réacteurs tels que les SMR, fourniront de nouveaux champs détude pour la LIBS.

\section{RÉFÉRENCES}

[1] D.L'Hermite, J.-B.Sirven, Techniques de l'ingénieur, 2870 v1 (2015)

[2] J. Wu et al., J. Phys. D: Appl. Phys. 53, 023001 (2020)

[3] S. Legnaioli et al., Anal. Methods 12, 1014 (2020)

[4] J.-C. Brachet et al., J. Nucl. Mat. 488, 267 (2017)

[5] C. Maury et al., Spectrochim. Acta Part B 82, 28 (2013)

[6] D. L'Hermite et al., Journées LIBS 2015, Verneuil en Halatte, France (2015). HAL Id : cea-02489576

\section{W) Femto Easy}

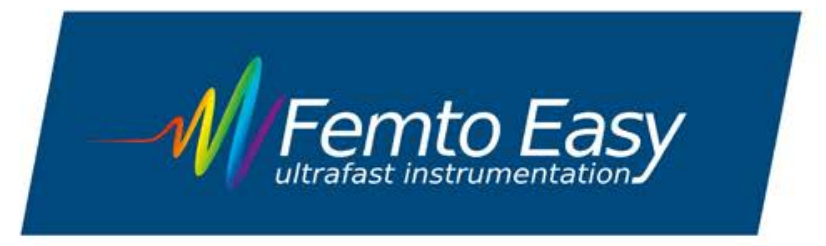

\section{Instrumentation innovante pour lasers ultrarapides}

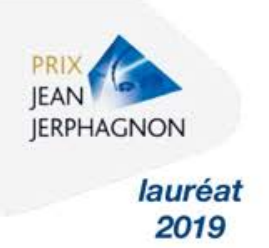

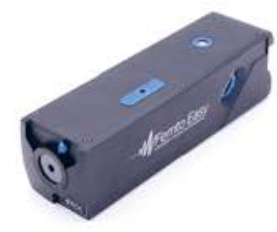

Autocorrélateur mono-coup et multi-coup

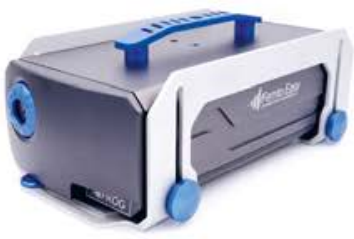

FROG

mono-coup et multi-coup

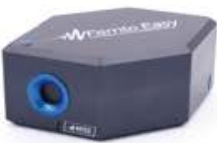

Spectromètre imageur compact
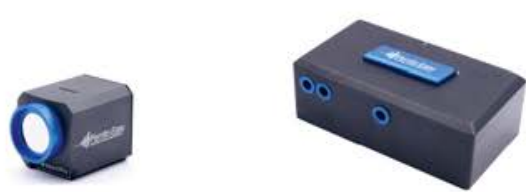

\section{Analyseur de faisceau \\ (tous types de} lasers)
Module de conversion de fréquence 